\title{
FDA narrows drug label usage
}

The US Food and Drug Administration (FDA) has altered the usage labels on two cancer drugs on the basis of a re-evaluation of clinical data.

The agency introduced the change last month after its Oncologic Drugs Advisory Committee (ODAC) recommended that the drugs Erbitux and Vectibix - approved in 2004 and 2006, respectively, for patients with advanced-stage colorectal cancer - should now be prescribed only to individuals with a certain gene variant. To reach the decision, the agency reviewed seven randomized clinical trials, all of which showed that only the $60 \%$ or so of patients whose tumours harbour the non-mutated or 'wild-type' form of a gene called $K-R A S$ responded positively to the drugs.

Ideally, the FDA would want to do these kinds of geneticmarker tests for patient response in a well-designed, forward-looking experiment, says ODAC consultant Richard Simon, chief of the National Cancer Institute's biometric research branch in Rockville, Maryland. "But cancer biology is very complex, and I think we're going to find that it's not always possible to have [all the answers] figured out beforehand."

Erbitux is made by ImClone Systems and marketed by Bristol-Myers Squibb, both based in New York. Vectibix is made by Amgen, headquartered in Thousand Oaks, California.

Analysts and scientists are split on whether to expect after-the-fact label adjustments for other drugs. "The FDA will certainly become more open to these types of changes over time," says Bruce Booth, a pharmaceutical analyst with Atlas Venture in Boston, Massachusetts.

ODAC member Gary Lyman of the Duke Comprehensive Cancer Center in Durham, North Carolina, says that revisiting drug efficacies could become so widespread that the FDA should at least encourage companies to bank tissues from finished trials for unanticipated post-hoc tests.

David Reese, Amgen's executive director of oncology, says that some of the retrospective analyses would not have been possible had the company not banked tissues. "Obtaining tissues and having [them] available in the future is really critical," he says.

But David Harrington, a statistician at the Dana-Farber Cancer Institute in Boston, Massachusetts, and another ODAC member, says future calls for retrospective analyses will probably be considered on a case-by-case basis. "It was the striking nature of the data that led to the recommendation" to change Erbitux and Vectibix labelling, he says, "and it's unlikely to happen very often in the future".

Indeed, many argue that the conditions surrounding the label change were unique and won't set a precedent. The American Society of Clinical Oncology and the National Comprehensive Cancer Network had already recommended that doctors test for $K-R A S$ variants before treatment. Moreover, the drug companies themselves had asked for restricted use after learning about the $K$-RAS results. In announcing the change, the FDA affirmed that both drugs are "not recommended for the treatment of colorectal cancer with $[K-R A S]$ mutations".

Doctors are now advised but not compelled - to test for $K-R A S$ before administering the drugs, notes Stephen Little, chief executive of DxS, a diagnostics company in Manchester, UK, that markets a $K-R A S$ mutation test kit. He says that most drug companies now conduct diagnostic screening during clinical development.

In Europe, Erbitux's label changed more than a year ago to reflect the $K-R A S$ status, and Vectibix won approval only for those patients with non-mutated $K-R A S$. "Europe has been more inclined to look at retrospective data if otherwise those data are robust and reflect the general population in that trial," says Hagop Youssoufian, senior vice-president of clinical research and development at ImClone in Branchburg, New Jersey.

Both drugs are currently being tested for use in combination with chemotherapy; data on this will be presented next month at a joint meeting in Berlin of the European Cancer Organisation and the European Society for Medical Oncology. These tests include two phase III trials, of roughly 1,200 patients each, that show Vectibix in combination with chemotherapy kept tumours in check for significantly longer in cancer patients with wild-type $K-R A S$, and a similar trial - details of which are not yet public - involving Erbitux.

Elie Dolgin 